\title{
Electrochemical Properties of Binary Electrolytes for Lithium-sulfur Batteries
}

\author{
Hyung Sun Kim* and Chang-Sik Jeong \\ Energy Storage Research Center, Korea Institute of Science and Technology (KIST), P.O. Box 131, \\ Cheongryang,Seoul 130-650,Korea. E-mail: kimhs@kist.re.kr \\ Received July 23, 2011, Accepted August 17, 2011
}

\begin{abstract}
The electrochemical properties of lithium-sulfur batteries with binary electrolytes based on DME and DOL, TEGDME and DOL mixed solvent containing $\mathrm{LiClO}_{4}$, LiTFSI, and LiTF salts were investigated. The ionic conductivity of $1 \mathrm{M} \mathrm{LiTFSI} \mathrm{and} \mathrm{LiClO}_{4}$ electrolytes based on TEGDME and DOL increased as the volume ratio of DOL solvent increased, because DOL effectively reduces the viscosity of the above electrolytes medium under the same salts concentration. The first discharge capacity of lithium-sulfur batteries in the DME and DOL-based electrolyte followed this order: LiTFSI $(1,000 \mathrm{mAh} / \mathrm{g})>\operatorname{LiTF}(850 \mathrm{mAh} / \mathrm{g})>\mathrm{LiClO}_{4}(750 \mathrm{mAh} /$ g). In case of the electrolyte based on TEGDME and DOL, the first discharge capacity of batteries followed this order: $\mathrm{LiClO}_{4}(1,030 \mathrm{mAh} / \mathrm{g})>\operatorname{LiTF}(770 \mathrm{mAh} / \mathrm{g})>$ LiTFSI $(750 \mathrm{mAh} / \mathrm{g})$. The cyclic efficiency of lithium-sulfur batteries at $1 \mathrm{M} \mathrm{LiClO}_{4}$ electrolytes is higher than that of batteries at other lithium salts-based electrolytes. Lithium-sulfur battery showed discharge capacity of $550 \mathrm{mAh} / \mathrm{g}$ until 20 cycles at all electrolytes based on DME and DOL solvent. By contrast, the discharge capacity of batteries was about $450 \mathrm{mAh} / \mathrm{g}$ at $1 \mathrm{M}$ LiTFSI and LiTF electrolytes based on TEGDME and DOL solvent after 20 cycles.
\end{abstract}

Key Words : Lithium-sulfur batteries, Binary electrolytes, Cyclic performances

\section{Introduction}

Lithium-sulfur batteries are considered to be very attractive candidates for power sources because of their high energy density. ${ }^{1-3}$ Elemental sulfur has the highest theoretical specific capacity of $1,672 \mathrm{mAh} / \mathrm{g}$ and energy density of $2600 \mathrm{Wh} / \mathrm{kg}$ when compared with other cathode materials. The development of lithium-sulfur batteries has been performed widely by many research groups to take advantage of their high energy density. ${ }^{4-6}$ Although lithium-sulfur batteries have many advantages, the utilization of sulfur cathode and the cyclic stability of these batteries still remain to be a severe problem due to the loss of sulfur active material and dendritic formation of lithium anode surface in the repeated cycles. The sulfur cathode forms various lithium polysulfides $\left(\mathrm{Li}_{2} \mathrm{~S}_{\mathrm{n}}, \mathrm{n}=3-8\right)$ as a result of reduction during the discharge process. Due to their high solubility in the organic electrolyte, polysulfide anions are easy to dissolve into the electrolyte and actively react with lithium anode. This shuttle phenomenon hinders the high utilization of sulfur cathode material and cyclic efficiency of lithiumsulfur batteries during the cycling. Therefore, the advantages of lithium-sulfur batteries should be limited, unless the electrolyte is stable. It has been reported that a mixed solvent of ether and cyclic solvent has high ionic conductivity, electrochemical stability, chemical compatibility against lithium metal, and safety. ${ }^{7,8}$ The effects of solvent component on the electrochemical properties of lithium-sulfur batteries have been studied by many researchers. ${ }^{9-15}$ According to the previous studies, 1, 2-dimethoxy ethane (DME), 1, 3-dioxolane (DOL), tetrahydrofuran, tetra(ethylene glycol)dimethyl ether (TEGDME), tri(ethylene glycol)dimethyl ether, and diglyme organic solvents can be used in a lithiumsulfur batteries. The electrolytes usually can be used by mixed solvents and, thus optimization of the solvent composition is very important to meet the previous requirements of the electrolyte.

In this work, we prepared electrolyte systems based on a binary solvent mixture of DME and DOL, TEGDME and DOL solvents with different volume ratios containing various lithium salts. We investigated the ionic conductivity and viscosity of these binary electrolytes and the charge/discharge properties of lithium-sulfur batteries in the optimized electrolytes.

\section{Experimental}

The lithium salts used were $\mathrm{LiCF}_{3} \mathrm{SO}_{3}(\mathrm{LiTF}), \mathrm{LiN}\left(\mathrm{CF}_{3} \mathrm{SO}_{2}\right)_{2}$ (LiTFSI), and $\mathrm{LiClO}_{4}$. These lithium salts were purchased from Aldrich and used without pretreatment. The electrolytes used were each $1 \mathrm{M}$ lithium salts in solvent of binary mixture of DME (Aldrich, 98\%) and DOL (Aldrich, 98\%), TEGDME (Aldrich, 98\%) and DOL with different volume ratios. The volume ratios of DME and DOL, TEGDME and DOL were varied as 67\%:33\%, 50\%:50\%, and 33\%:67\%, respectively. The sulfur cathode consisted of $70 \mathrm{wt} \%$ of elemental sulfur as an active material (Aldrich, 98\%), 15 wt. $\%$ of Super P as a conductor, 15 wt. $\%$ of poly(vinylidene fluoride) with a 1-methyl-2-pyrrolidinone organic solvent as a binder. The mixed slurry was cast on an aluminum foil, dried at $80^{\circ} \mathrm{C}$ in a vacuum oven for $24 \mathrm{~h}$ to remove residual organic solvent, and pressed using rolling machine. The ionic conductivity and viscosity of electrolytes were measured by means of conductivity meter (Metrohm 
856, Tiamo) and viscometer (SV-10, AND) in a dry room (dew point: $-60^{\circ} \mathrm{C}$ ) at room temperature. In order to measure the electrochemical properties, the sulfur cathode was assembled along with a lithium foil anode using CR 2032 coin cells in the dry room and a microporous polypropylene separator (Celgard 2400) was used. The ac impedance measurements were performed over the frequency range of 1 $\mathrm{MHz}$ to $0.1 \mathrm{~Hz}$ using a Solartron 1260 frequency response analyzer coupled with a 1286 electrochemical interface. The cycle test of lithium-sulfur batteries was conducted in the voltage range from 1.5 to $2.8 \mathrm{~V} v s$. $\mathrm{Li} / \mathrm{Li}^{+}$by constant current method using a Maccor cycle system (S 4000, USA).

\section{Results and Discussion}

Figure 1(a-c) shows the change of ionic conductivity of the DME and DOL, TEGDME and DOL-based binary electrolytes with different volume ratios containing various lithium salts. The highest ionic conductivity of $11 \mathrm{mS} / \mathrm{cm}$ is observed at $1 \mathrm{M}$ LiTFSI electrolyte in the DME:DOL of $50 \%: 50 \%$ volume ratio. The ionic conductivity decreased at the higher portion of DOL. The results suggested that DME could provide better capability to dissociate lithium salts than DOL. The ionic conductivity increased as the volume ratio of DOL solvent increased at $1 \mathrm{M}$ LiTFSI and $\mathrm{LiClO}_{4}$ electrolytes based on TEGDME and DOL. In case of $1 \mathrm{M}$ LiTF electrolyte, the ionic conductivity keeps almost constant value of $2 \mathrm{mS} / \mathrm{cm}$ irrespective of the concentration of DOL in DME or TEGDME-based solvents, respectively. The ionic conductivity of electrolyte is generally governed by the degree of ionic concentration and mobility. Therefore, the high ionic conductivity of electrolyte could be explained by the increased ionic mobility under the same salt concentration. The high ionic mobility would be responsible for the relatively low viscosity of the conducting medium. The viscosity of binary electrolyte as a mixing ratio of DME and DOL, TEGDME and DOL at each $1 \mathrm{M}$ lithium salts is presented in Figure 2(a-c). The viscosity of binary electrolytes based on TEGDME solvent decreased as the concentration of DOL solvent increased. DOL solvent is known for an effective solvent to reduce the viscosity of the electrolyte medium. ${ }^{16}$ By contrast, the viscosity of electrolyte based on DME solvent maintains constant value of about $1 \mathrm{cP}$ irrespective of the concentration of DOL solvent. This effect of DOL solvent on the viscosity of electrolyte could be attributed to the higher viscosity of TEGDME solvent compared to the viscosity of DME solvent. Figure 3 shows the initial discharge/charge voltage curves of lithium-sulfur batteries in DME and DOL(a), TEGDME and DOL(b)based binary electrolytes containing $1 \mathrm{M} \mathrm{LiClO}_{4}, 1 \mathrm{M}$ LiTFSI, and $1 \mathrm{M}$ LiTF salts at $0.1 \mathrm{C}$ rate, respectively. The volume ratio of DME and DOL, TEGDME and DOL is 50\%:50\%. There are two distinct discharge voltage plateau derived from the reduction of elemental sulfur to lithium polysulfide $\left(\mathrm{Li}_{2} \mathrm{~S}_{8}, \mathrm{Li}_{2} \mathrm{~S}_{6}, \mathrm{Li}_{2} \mathrm{~S}_{4}\right.$, and $\left.\mathrm{Li}_{2} \mathrm{~S}_{3}\right)$ at higher voltage, and reduction of lithium polysulfide to a solid reduction product, such as $\mathrm{Li}_{2} \mathrm{~S}_{2}$ and $\mathrm{Li}_{2} \mathrm{~S}$ at lower voltage in all
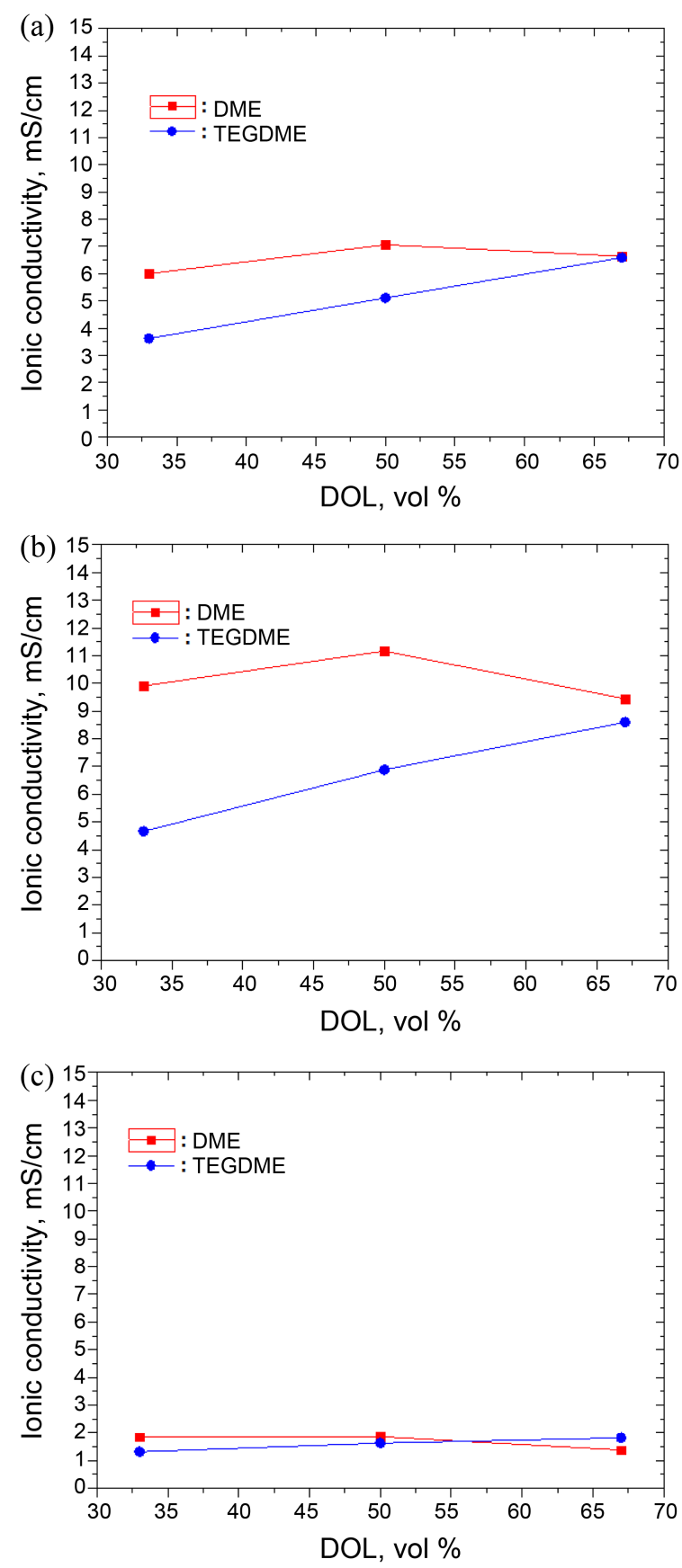

Figure 1. The ionic conductivity of the DME and DOL, TEGDME and DOL-based binary electrolytes at different volume ratios containing $1 \mathrm{M} \mathrm{LiClO}_{4}$ (a), 1 M LiTFSI (b), and $1 \mathrm{M} \mathrm{LiTF}$ (c) salts.

electrolytes. Lithium polysulfides, which are generated intermediate products during the discharge process, may be dissolved in the binary electrolyte. They diffuse into the electrolyte through separator and react directly with the lithium electrode to regenerate lower forms of polysulfide. These species diffuse back to the sulfur cathode to generate higher forms of polysulfide again, thus creating a shuttle mechanism. ${ }^{17-19}$ This shuttle reaction reduces the cyclic efficiency of lithium-sulfur batteries. The low cyclic efficiency at the end of charge process is the main factor limiting sulfur utilization during the cycling. This work focused on 

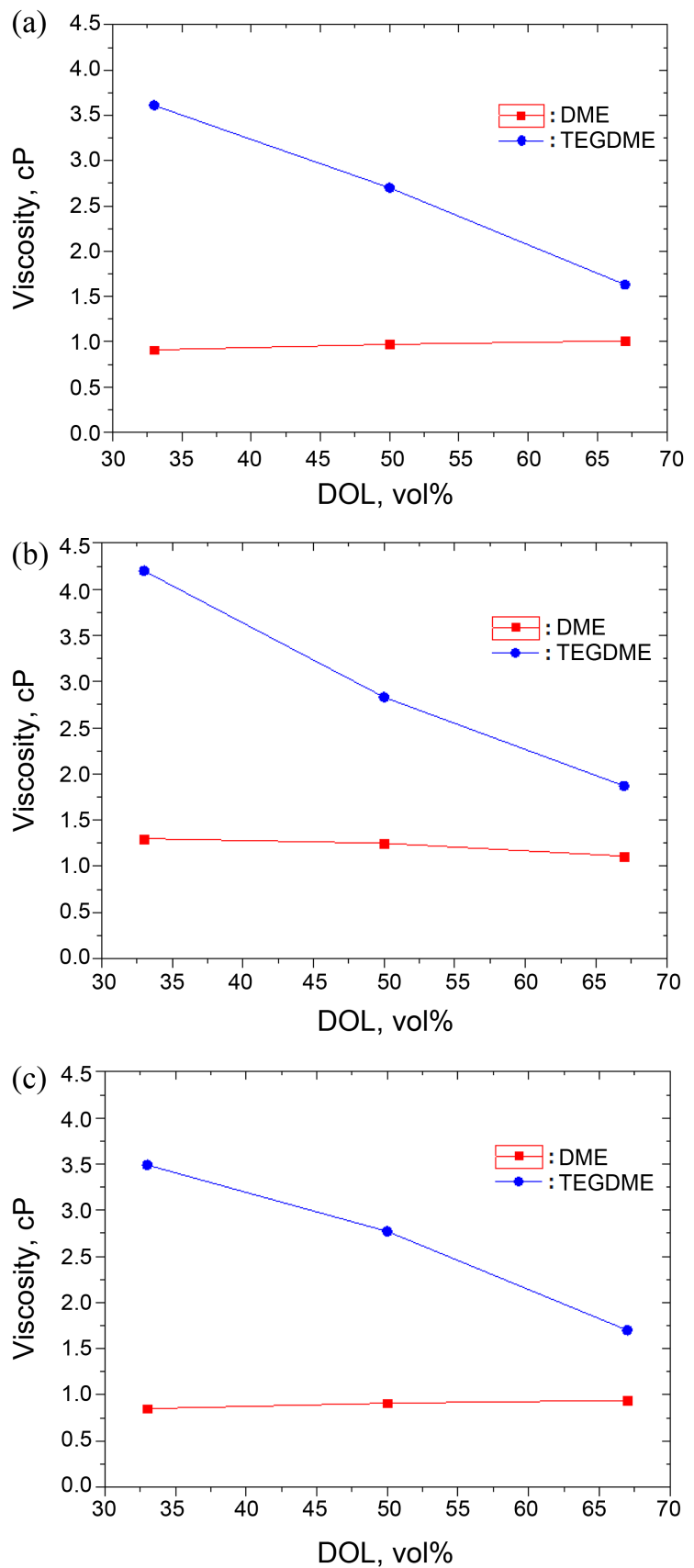

Figure 2. The viscosity of the DME and DOL, TEGDME and DOL-based binary electrolytes at different volume ratios containing $1 \mathrm{M} \mathrm{LiClO}_{4}$ (a), $1 \mathrm{M} \mathrm{LiTFSI} \mathrm{(b),} \mathrm{and} 1 \mathrm{M} \mathrm{LiTF}$ (c) salts.

the increase of the cyclic efficiency through the optimized electrolyte formulation to attain higher specific capacity. There is no difference in the length of the first plateau with the change of lithium salts in the DME and DOL based on electrolyte during the discharge. The first discharge capacity of the test batteries followed this order: LiTFSI $(1,000 \mathrm{mAh} /$ g) $>\operatorname{LiTF}(900 \mathrm{mAh} / \mathrm{g})>\mathrm{LiClO}_{4}(750 \mathrm{mAh} / \mathrm{g})$. However, the patterns of charge voltage curves are seen to be highly dependent on the lithium salts. The width of the higher potential plateau in $1 \mathrm{M}$ LiTFSI and $1 \mathrm{M}$ LiTF electrolytes were much longer due to the strong shuttle reaction com-


Figure 3. The initial discharge/charge voltage curves of lithiumsulfur batteries in DME and DOL (a), TEGDME and DOL (b)based binary electrolytes containing $1 \mathrm{M} \mathrm{LiClO}_{4}, 1 \mathrm{M} \mathrm{LiTFSI}$, and 1 M LiTF salts.

pared with $1 \mathrm{M} \mathrm{LiClO}_{4}$ electrolyte at the first cycle. In case of the electrolyte based on TEGDME and DOL, the discharge capacity of the test batteries followed this order: $\mathrm{LiClO}_{4}(1,030 \mathrm{mAh} / \mathrm{g})>\operatorname{LiTF}(770 \mathrm{mAh} / \mathrm{g})>\operatorname{LiTFSI}(750$ $\mathrm{mAh} / \mathrm{g}$ ). The discharge/charge voltage curves of these electrolytes also show different patterns when compared with electrolytes based on DME and DOL solvent. The charge efficiency at the end of charge voltage of these electrolytes, especially $1 \mathrm{M}$ LiTF electrolyte, is higher than that of electrolyte based on DME and DOL solvent due to the decreased shuttle phenomena. This hindered shuttle reaction may be attributed to the low diffusion of lithium polysulfide in the increased viscosity of electrolyte by TEGDME solvent. The charge efficiency of electrolyte based on $1 \mathrm{M}$ $\mathrm{LiClO}_{4}$ was almost independent on the types of solvents. Meanwhile, the charge efficiency of $1 \mathrm{M}$ LiTFSI electrolyte based on TEGDME and DOL decreased. In order to have a better understanding to the effect of electrolyte composition on the electrochemical performance, the impedance properties of lithium-sulfur batteries were investigated. The ac voltage used during the measurements was $10 \mathrm{mV}$. The impedance spectrum of lithium-sulfur batteries in Figure 4 show the lines intercepting the real part at high frequency, corresponding to the resistance of the electrolyte. Two 



Figure 4. Electrochemical impedance spectrum of lithium-sulfur battery before (a) and after the $1^{\text {st }}$ discharge (b) in TEGDME and DOL-based binary electrolytes containing $1 \mathrm{M} \mathrm{LiClO}_{4}, 1 \mathrm{M} \mathrm{LiTFSI}$, and $1 \mathrm{M}$ LiTF salts.

obvious semicircles were also found after the first discharge. The first semicircle at high frequency represents resistance of the solid state interface layer formed on the surface of the electrode. The faradic charge transfer resistance is corresponded to the diameter of the second semicircle at the middle frequency. The decrease in the diameter of the semicircles could improve the interfacial properties between and electrode and electrolyte. The results suggested that the lower charge transfer resistance could lead to the inhibition of polysulfide shuttle reaction and thus increase the cyclic efficiency of lithium-sulfur batteries. Figure 5 shows the cyclic performances of lithium-sulfur batteries in DME and DOL(a), TEGDME and DOL(b)-based binary electrolytes containing $1 \mathrm{M} \mathrm{LiClO}_{4}, 1 \mathrm{M} \mathrm{LiTFSI}$, and $1 \mathrm{M} \mathrm{LiTF}$ salts at $0.1 \mathrm{C}$ rate, respectively. The discharge capacity of the battery in the electrolyte based on DME and DOL rapidly decreased for the initial 5 cycles and maintained about $550 \mathrm{mAh} / \mathrm{g}$ without a further capacity loss until 20 cycles at all electrolytes. In case of the electrolyte based on TEGDME and DOL, the discharge capacity of the battery also decreased for the initial 5 cycles and maintained about $450 \mathrm{mAh} / \mathrm{g}$ until 20 cycles at $1 \mathrm{M} \mathrm{LiTF}$ and $1 \mathrm{M}$ LiTFSI electrolytes. The cyclic performance of the battery with $1 \mathrm{M} \mathrm{LiClO}_{4}$ electrolyte gradually decreased as cycle proceeded. The higher
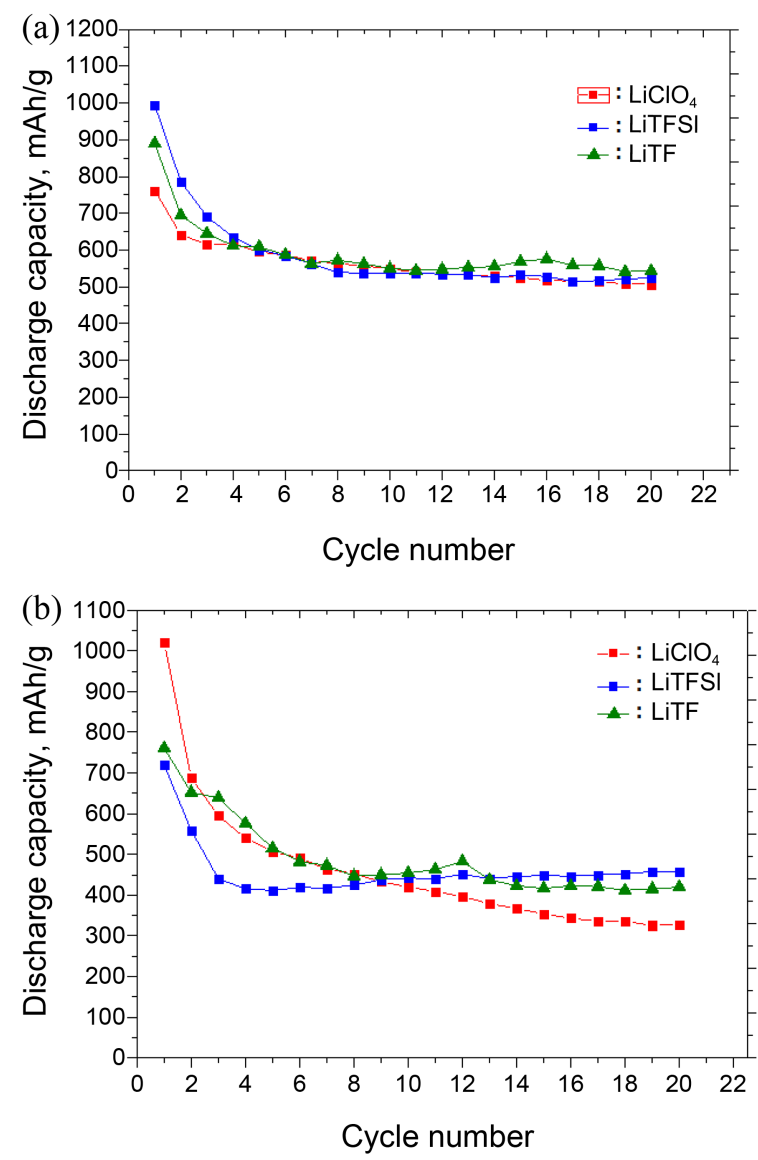

Figure 5. The cyclic performances of lithium-sulfur batteries in DME and DOL (a), TEGDME and DOL (b)-based binary electrolytes containing $1 \mathrm{M} \mathrm{LiClO}_{4}, 1 \mathrm{M} \mathrm{LiTFSI}$, and $1 \mathrm{M} \mathrm{LiTF}$ salts.

discharge capacity of the battery in DME and DOL-based electrolyte could be explained by good surroundings for the redox reaction. Figure 6 shows the impedance spectrum of lithium-sulfur batteries in DME and DOL(a), TEGDME and DOL(b) electrolytes after 20 cycles, respectively. It could be seen that the diameters of two semicircles, which are corresponded to the passivation layer and charge transfer resistance, were larger in electrolyte based on TEGDME and DOL than the electrolyte based on DME and DOL. The passivation layer in TEGDME and DOL-based electrolytes could be formed easily compared to DME and DOL-based electrolytes due to the higher viscosity, which restricted the lithium ions moving into inside of the cathode material, hence the redox reaction on the surface cathode decreased in the TEGDME and DOL-based electrolytes.

\section{Conclusion}

It is shown that the ionic conductivity of the binary electrolyte based on DME and DOL, TEGDME and DOL depends on the types of the added salts such as $\mathrm{LiClO}_{4}$, LITFSI, and LiTF. In addition, the ionic conductivity of the electrolyte based on DME and DOL solvent is higher than that of electrolyte based on TEGDME and DOL at the low concentration of DOL solvent. We have related this effect to 

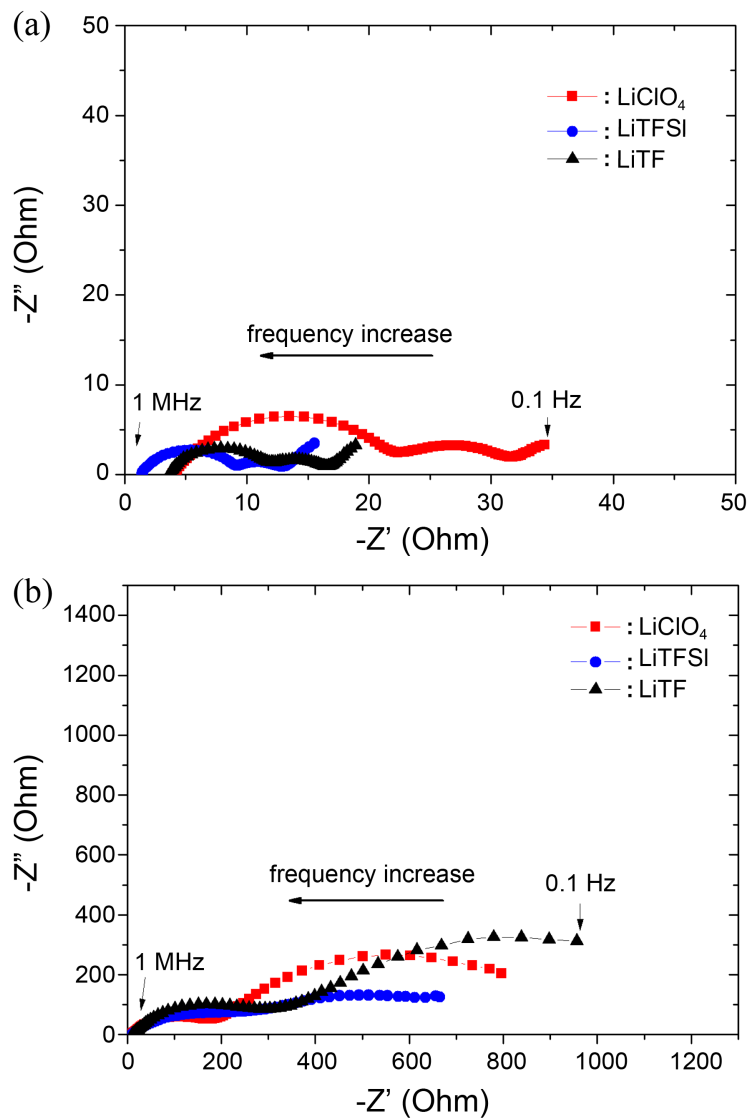

Figure 6. Electrochemical impedance spectrum of lithium-sulfur batteries after 20 cycles in DME and DOL (a), TEGDME and DOL (b)-based binary electrolytes containing $1 \mathrm{M} \mathrm{LiClO}_{4}, 1 \mathrm{M}$ LiTFSI, and $1 \mathrm{M}$ LiTF salts.

the increased viscosity of electrolyte due to the high concentration of TEGDME solvent. The ionic conductivity of the electrolyte based on TEGDME solvent is well correlated with the viscosity of the electrolyte phase. The cyclic efficiency of lithium-sulfur batteries is higher in $1 \mathrm{M} \mathrm{LiClO}_{4}$ electrolyte than that of batteries in other salts-based electrolytes. However, lithium-sulfur batteries maintained the discharge capacity of $550 \mathrm{mAh} / \mathrm{g}$ until 20 cycles without a further capacity loss at all electrolytes based on DME and DOL despite the low cyclic efficiency. In case of the electro- lyte based on TEGDME and DOL, the discharge capacity of batteries was about $450 \mathrm{mAh} / \mathrm{g}$ at $1 \mathrm{M}$ LiTFSI and LiTF electrolyte even after 20 cycles, but decreased gradually at 1 $\mathrm{M} \mathrm{LiClO}_{4}$ electrolyte as cycle proceeded.

Acknowledgments. This study was funded by "The Development of the Next Generation Lithium Metal Battery for the Full EV Project" of the Ministry of Knowledge Economy of Korea.

\section{References}

1. Choi, Y.; Kim, K.; Ahn, H.; Ahn, J. J. Alloys and Compounds 2008, 449, 313

2. Zhu, X.; Wen, Z.; Gu, Z.; Lin, Z. J. Power Sources 2005, 139, 269.

3. Akridge, J.; Mikhaylik, Y.; White, N. Solid State Ionics 2004, 175, 243.

4. Wang, J.; Yang, J.; Xie, J.; Xu, N.; Li Y. Electrochemistry Communications 2002, 4, 499.

5. Marmorstein, D.; Yu, T.; Striebel, K.; McLarnon, F.; Hou, J.; Cairns, E. J. Power Sources 2001, 89, 219.

6. Kim, S.; Jung, Y.; Park, S. Electrochimica Acta 2007, 52, 2166.

7. Kim, S.; Jung, Y.; Lim, H. Electrochimica Acta 2004, 50, 889.

8. Yamin, H.; Gorenshtein, A.; Penciner, J.; Sternberg, Y.; Peled, E. J. Electrochemical Society 1988, 135, 1045.

9. Rhu, H.; Ahn, H.; Kim, K.; Ahn, J.; Cho, K.; Nam, T. J. Power Sources 2006, 163, 201.

10. Cheon, S.; Ko, K.; Cho, J.; Kim, S.; Chin, E.; K, H. J. Electrochemical Society 2003, 150, A796.

11. Rhu, H.; Ahn, H.; Kim, K.; Ahn, J.; Lee, J. J. Power Sources 2006, 153,360 .

12. Rhu, H.; Ahn, H.; Kim, K.; Ahn, J.; Lee, J.; Cairns, E. J. Power Sources 2005, 140, 365.

13. Jin, B.; Kim, J.; Gu, H. J. Power Sources 2003, 117, 148.

14. Trofimov, B.; Markova, M.; Morzova, L.; Prozorova, G.; Korzhova, S.; Cho, M.; Annenkov, V.; Mikhaleva, A. Electrochimica Acta 2011, 56, 2458.

15. Choi, J.; Kim, J.; Cheruvally, G.; Ahn, J.; Ahn, H.; Kim, K. Electrochimica Acta 2007, 52, 2075.

16. Chang, D.; Lee, S.; Kim, S.; Kim, H. J. Power Sources 2002, 112 , 452.

17. Rauh, R.; Ahraham, K.; Pearson, G.; Suprenant, J.; Brummer, S. J. Electrochemical Society 1979, 126, 523.

18. Peled, E., Gorenshtein, A.; Segal, M.; Sternberg, Y. J. Power Sources 1989, 26, 269.

19. Peled, E.; Sternberg, Y.; Gorenshtein, A.; Lavi, Y. J. Electrochemical Society 1989, 136, 1621. 